\title{
Smart lighting system ISO/IEC/IEEE 21451 compatible
}

\author{
J. Higuera, Member, IEEE, W. Hertog, M. Perálvarez, J. Polo, and J. Carreras
}

\begin{abstract}
Smart lighting systems go far beyond merely replacing lamps. These modern systems are now able to reproduce arbitrary spectra, color temperatures, intensities and pivot on smart sensors and actuators incorporating Information and Communication Technologies (ICT). This work presents an interoperable smart lighting solution that combines heterogeneous lighting technologies enabling intelligent functions. The system can shift light intensity to increase visual comfort and it is oriented towards human centric lighting studies. Moreover, this system follows the guidelines defined by the ISO/IEC/IEEE 21451 standards and ZigBee Light Link (ZLL) and also, it includes an additional transducer signal treatment service for artificial intelligence algorithms. Finally, a REST application allows us to test the interoperability and visualize energy consumption and savings in an office room.
\end{abstract}

Index Terms - Internet of Things (IoT), interoperability, ISO/IEC/IEEE 21451, NCAP, REST web service, smart lighting, TIM, ZigBee Light Link (ZLL).

\section{INTRODUCTION}

S SMART lighting is a buzzword that defines heterogeneous lighting technologies: High-intensity discharge lamps (HID), fluorescent, solid state LED and OLED luminaires [1], composed of numerous smart sensors and actuators, and with the possibility of incorporating a wide set of capabilities and connectivity interfaces. The most important intelligent features are related to enabling advanced functions such as adjustable spectral reproduction [2], color tunable-lighting [3], and adaptive dimming [4], combining energy efficiency with the real needs of the illuminated space

Manuscript received October 25, 2014. "This project has received funding front the European Union Seventh Framework Programme (FP7/2007-2013) under grant agreement no 619912”. Moreover, this investigation has been supported by efficient lighting and HVAC control system for buildings (ECOE). Spanish Ministry of Economy and Competitiveness subprogram Innpacto 2011

J. Higuera is with the Catalonia Institute for Energy Research (IREC), Barcelona, Spain, (e-mail: jehiguera@irec.cat).

W. Hertog is with the Catalonia Institute for Energy Research (IREC), Barcelona, Spain, (e-mail: whertog@irec.cat).

M. Perálvarez is with the Catalonia Institute for Energy Research (IREC), Barcelona, Spain, (e-mail: mperalvarez@irec.cat).

J. Carreras is with the Catalonia Institute for Energy Research (IREC), Barcelona, Spain, (e-mail: jcarreras@irec.cat).

J. Polo is with the Universitat Politècnica de Catalunya-BarcelonaTech (UPC), ISI group, Castelldefels, Barcelona, Spain, (e-mail: jose.polo@upc.edu). while taking into account available natural lighting. With the advent of wireless communication technologies, smart lighting systems consist of different wired and wireless interfaces oriented to increment the connectivity in smart grid systems [5] and Building Management Systems (BMS) [6]. These systems allow us to control and monitor modern and heterogeneous electrical equipments such as ventilation and lighting devices. The main interfaces conceived for wired lighting systems are Digital Addressable Lighting Interface (DALI), Power Line Communications (PLC), Digital Multiplex (DMX512) and KNX for intelligence buildings.

The most important wireless physical interfaces for distributed intelligent devices are IEEE $802.11 \mathrm{a} / \mathrm{b} / \mathrm{g} / \mathrm{n} / \mathrm{ac}$ (Wi-Fi), 802.15.1 (Bluetooth), IEEE 802.15.4 (ZigBee, 6loWPAN, ISA100, WirelessHart, MiWi) [7] and ISO/IEC 18000 (RFID) compatible for automation applications [8].

Now modern smart lighting systems contain wired and wireless interfaces, and they can be harmonized in a set of ISO/IEC/IEEE 21451 standards [9]-[12]. This harmonization covers Transducer Electronic Datasheets (TEDS) formats. TEDS are different electronic data sheets stored in a non volatile flash memory. These formats define node capabilities and transducer channel configuration. In addition, ISO/IEC/IEEE 21451 standard covers standard syntactic messages, and logical transducer function services to be implemented in the Network Capable Application module (NCAP) and/or the Transducer Interface Module (TIM).

In its core, each NCAP is the coordinator node and the TIM module will be integrated in the luminaries or different smart sensors. Both can be organized as a Wireless Sensor and Actuator Network (WSAN) [13].

The IEEE 21451 reference model enables the coexistence of smart lighting systems and also allows interoperability through web services [14] to exchange data among multiple heterogeneous networks in the new wave of human centric lighting applications[15],[16].

An interoperable smart lighting system that implements the IEEE 802.15.4 physical layer and ZigBee Light Link (ZLL) [17], plus ISO/IEC/IEEE 21451 as application layer, has been implemented in this study as a practical solution for communication between wireless lighting devices, colour sensors, occupancy detectors and illuminance sensors.

In order to demonstrate the use of Fuzzy Logic theories in smart lighting systems, a predictive algorithm adjusts the dimming level based on the occupancy level, work schedule and natural lighting available. A graphical user interface was 
designed in Matlab to run the fuzzy algorithm. Finally, all information is encapsulated in standard message structures to interact with the Representational State Transfer (REST) web service architecture [18]. The web service runs on a free platform that contains an open API intended to control intelligent devices for the Internet of Things (IoT).

The paper is organized as follows: the ISO/IEC/IEEE 21451 reference model (NCAP and WTIM) is presented in section II. Section III describes the hardware architecture of a smart luminaire and autonomous sensor nodes. Next, in section IV, the algorithm intended for smart lighting control is explained. The REST web service architecture and experimental results are presented in section V. Finally, in section VI conclusions and considerations for effective energy savings which can be achieved through the proposed solution are presented.

\section{ISO/IEC/IEEE 21451 REFERENCE MODEL}

The family of ISO/IEC/IEEE 21451 (formerly IEEE 1451) [19] aims to expand the syntactic interoperability through a standardized message format implemented using heterogeneous smart sensors and actuators. Each Transducer Interface Module (TIM) works in cooperation with a Network Capable Application module (NCAP) that is operating as coordinator node entity. The NCAP architecture is shown in Figure 1.

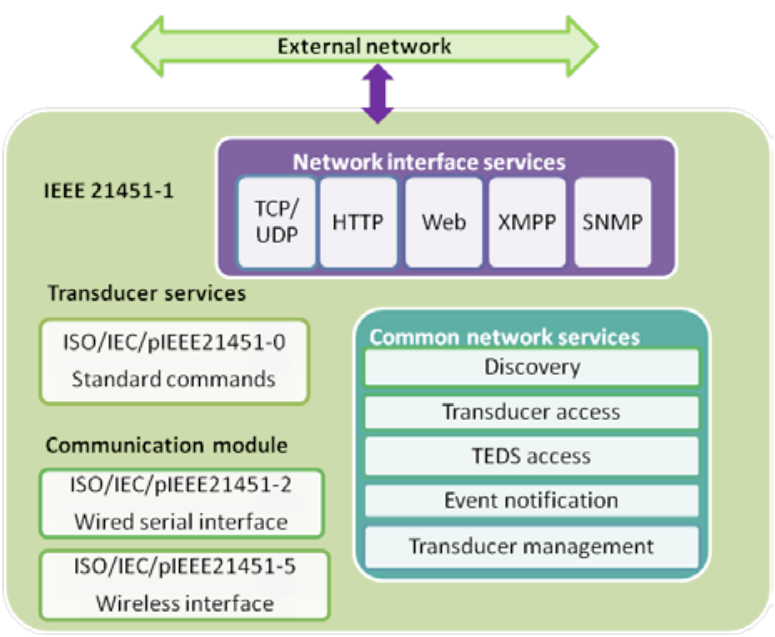

Fig. 1. NCAP architecture.

Each NCAP entity defines a group of logical interface formats. The first set of network interface services is intended for Representational State Transfer (REST) web services. These formats are suggested for smart sensors with Internet connectivity. Different logical network formats intended for this proposal are TCP/UDP, HTTP, Web Interface, Simple Network Management Protocol (SNMP) and eXtensible Messaging and Presence Protocol (XMPP) [20].

The second set of logical formats that runs in the NCAP is defined as common core network services ISO/IEC/IEEE21451-1. These services includes sub-classes for discovering, transducer data access, Transducer Electronic
Data Sheet (TEDS) access, event notification, and transducer management in order to address, map and control any TIM device.

The third set of formats in ISO/IEC/IEEE21451-0, formerly IEEE 1451.0, defines common control commands between the NCAP and the TIM device. In addition, physical communication capabilities are defined in ISO/IEC/IEEE 21451-2 standard for smart sensors working in wired networks while the ISO/IEC/pIEEE 21451-5 standard (formerly IEEE 1451.5) is intended for smart sensors working in wireless sensor networks (WSN), (WiFi, IEEE 802.15.4, Bluetooth) while ISO/IEC/IEEE 21451.7 was proposed for RFID devices.

The second part of the ISO/IEC/IEEE 21451 reference model is related to the Transducer Interface Module (TIM). As shown in Figure 2.

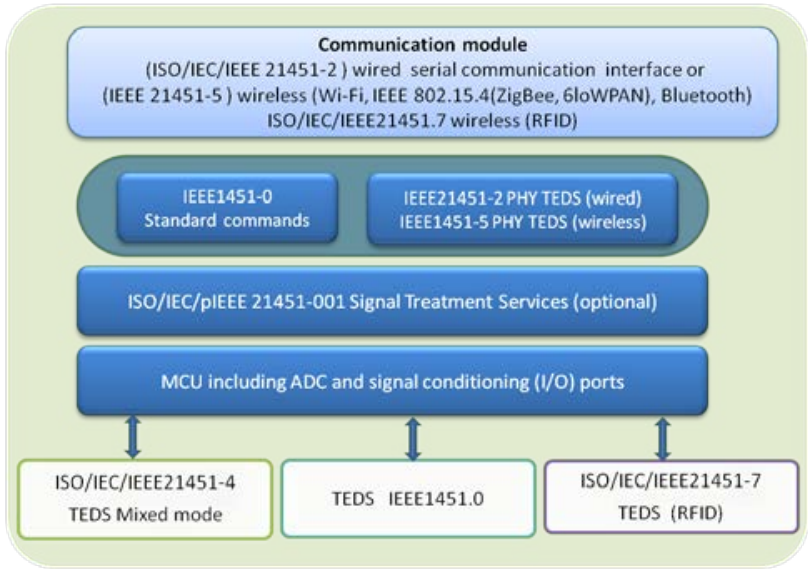

Fig. 2. TIM architecture.

Each TIM is an embedded physical device that implements a reduced set of logical services. The typical TIM hardware architecture contains an ultra low power microprocessor unit (MCU) and a non-volatile memory to store the node configuration in an electronic datasheet (TEDS). The IEEE 21451 reference model defines different standardized TEDS formats corresponding to the type of transducer. For physical transducer channels in a mixed mode interface the IEEE21451-4 standard TEDS is defined. For wired serial interfaces the IEEE 21451-2 standard TEDS is used. However, for RFID transducers in WSN, IEEE 21451-7 is the solution. In the case of transducers working in a WSN (sensors and actuators) that involves analog to digital conversion and signal conditioning, the IEEE 1451.5 physical TEDS was defined.

\section{HARDWARE ARCHITECTURE COMPLIANT WITH IEEE 21451}

The smart lighting system is deployed as an ecosystem of smart sensors located in walls, windows, the chilled beam system and the luminaires installed in the ceiling of the office. This section is focused on describing the luminaire architecture and the sensor node capabilities, including an energy harvesting module.

\section{A. Smart luminaire}

The platform designed is shown in Figure 3. It is based on 
Commercial Off-The-Shelf (COTS) digital sensors and actuators. The hardware architecture comprises six different sub-modules: a MCU processing unit, a wireless communication unit, a LED driver, flash memory, I2C/ADC channels (illuminance sensor, colour detection) and a digital input/output pin for digital occupancy sensor.

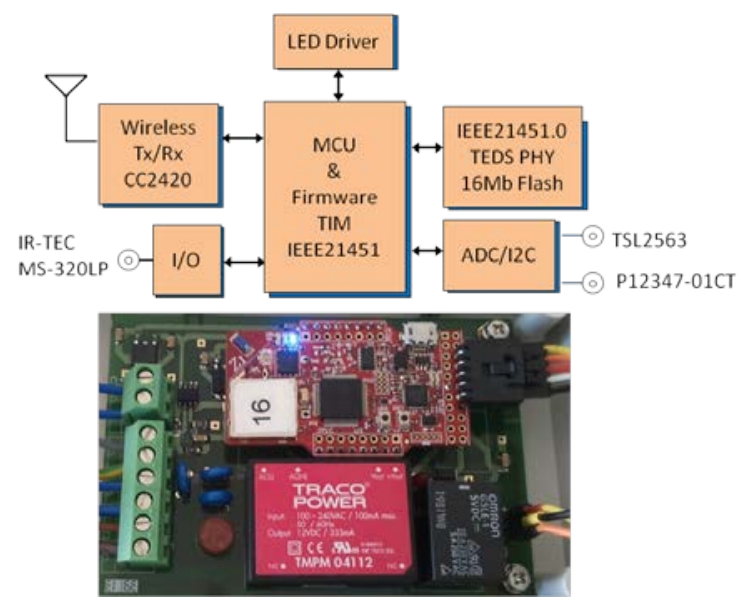

Fig. 3. Smart Luminaire block diagram and implemented system.

The MCU processing unit contains the MSP430 CPU and the firmware implements the ISO/IEC/IEEE 21451 TIM reference model. The decision-taking algorithm calculates the percentage of dimming to control the luminaire. One wireless transceiver is the core of the radio section. This device supports the IEEE 802.15.4 physical layer [21] and the ZigBee Light Link (ZLL) application layer [22].

The driver is composed of a solid state relay. This section uses the CPU I/O ports and DAC output and an amplifier for signal conditioning (1-10 VDC). Finally a circuit containing a BJT transistor and a protection diode manages the load. This sub-system is activated depending on occupancy detection or usage patterns related to studies on energy savings in lighting systems.

The flash memory stores metadata configuration related to the sensors channels compliant with the IEEE 21451.0 format and IEEE 21451.5 for the physical TEDS (IEEE 802.15.4 plus ZigBee Light Link).

Three different sensors are implemented in the sensing submodule: illuminance, occupancy and colour. For illuminance studies the TSL2563 sensor [23] containing two internal photodiodes, was selected due to high dynamic range (0 lx - $100.000 \mathrm{~lx}$ ) and capabilities to detect photopic and scotopic luminosity functions to measure the visual perception under different lighting conditions. The first photodiode captures illuminance in visible and infrared spectrum (300 nm - $1100 \mathrm{~nm}$ ) while the second photodiode captures only the infrared range of lighting $(600 \mathrm{~nm}-1100 \mathrm{~nm})$.

The occupancy detector is based on a passive infrared (PIR) digital IR-TEC MS-320LP sensor [24]. It contains dual elements for pyroelectric infrared sensing and the body includes a lens and an optical filter for passing infrared radiation.

For color measurements the P12347-01CT [25] was selected to adjust for color correlated temperature (CCT).

\section{B. Autonomous smart sensors}

For human centric lighting studies several autonomous sensors to measure illuminance were developed. All of them contain a photovoltaic harvester based on organic solar cells for indoor operation. Figure 4 shows the hardware architecture.

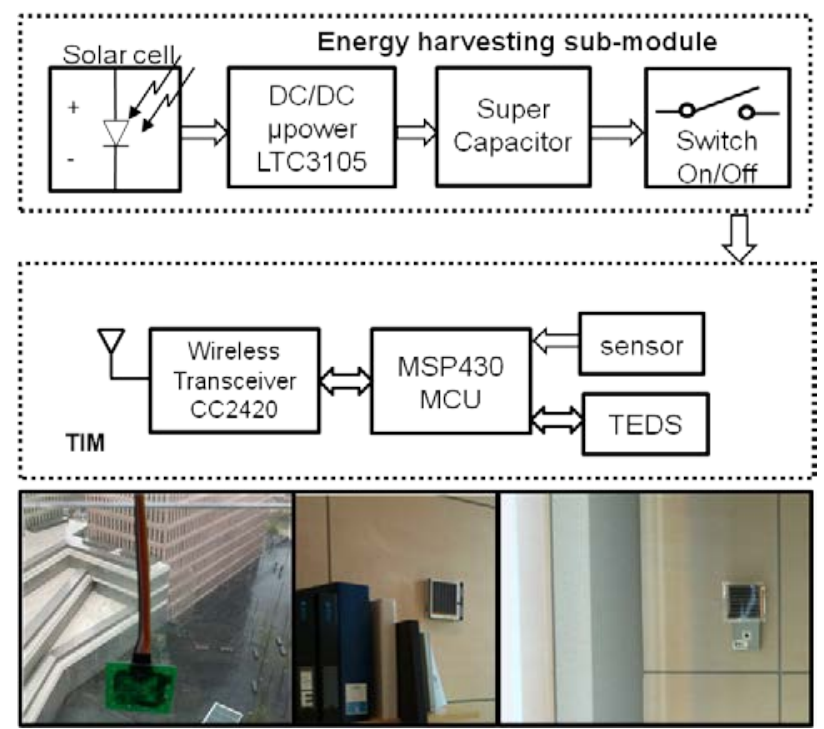

Fig. 4. Block diagram for smart sensor and their placement in the windows and office walls.

Each node contains an energy harvesting sub-module and a TIM unit. The energy harvesting unit contains organic and flexible solar cells, a direct current (DC/DC) micro-power regulator, super-capacitors and an ON/OFF switch to enable the device. The main TIM components are the MSP430 CPU, the external wireless transceiver and the flash memory to store the TEDS configuration. The nodes were located in the office near the windows and walls to measure illuminance values at the height of the working plane.

\section{Firmware implementation}

Figure 5 represents the operational states of the TIM and node capabilities in accordance with a finite-state machine model. The autonomous TIM wakes up when the harvester captures energy from the DSSC solar module due to an increase in daylight or artificial lighting levels.

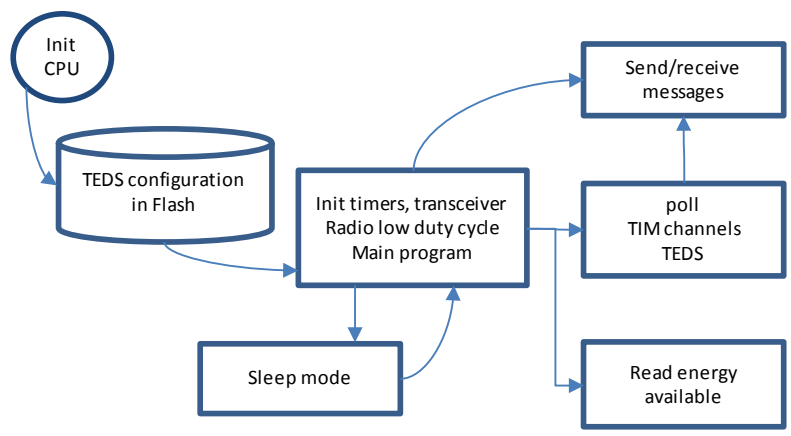

Fig. 5. TIM Finite State Machine. 
The first state is the initialization step (init) that occurs after an event related to power on or reset, time in which the TEDS configuration is loaded into the RAM memory. The second state is the active mode. In this mode, TIM polls each physical transducer channel and is already able to send or receive short messages. The third state is a sleep-mode that is used to reduce energy consumption. In this sleep state, the node waits for the expiration of a timer to wake up. The NCAP requests commands and the TIM responds with short messages corresponding to the command type.

After the initialization takes place, each TIM reads the TEDS calibration values for the physical channels. Once the program checks all the values, it saves the measured data in the RAM memory. The main loop runs once every five minutes. One loop runs the IEEE 21451 commands and sorts all arrays using standardized metadata formats. After the sorting procedure takes place, the program goes to active mode and starts the transceiver to send or receive messages. The mean values of physical channels are used because, occasionally, it can happen that incorrect values are measured due to powermanagement restrictions related to the node. These incorrect measured values have to be detected and filtered from true data.

In a time-frame of 2 milliseconds, the last measured data is stored in RAM order to be sent towards the NCAP. The coordinating NCAP is connected through a USB port from an industrial PC running a Windows 7 operating system and a Matlab application. Each message in the NCAP is encapsulated in short structures, in compliance with the IEEE 21451 standard. These message structures are sent to the TIM nodes. When the NCAP sends a message it waits for the TIM response during $500 \mathrm{~ms}$. If a response is not received, the coordinator waits five minutes before the process is repeated. Then, the IEEE 21451 packets are sent towards a virtual serial port to decode metadata in a JSON structure that runs in a Matlab graphical user interface before being sent out to the web service.

\section{TRANSDUCER SIGNAL SERVICES}

\section{A. Fuzzy logic algorithm}

Lighting control systems have been defined historically to minimize power consumption and/or increase visual comfort. Traditionally, these strategies are divided into four different approaches. The first, involves updating the lighting system with energy-efficient luminaires. The second approach, introduces occupancy sensors in the lighting system. In the third, the lighting system contains daylight harvesting and control. Finally in the fourth approach, the lighting system further includes motorized blinds. However, in our lighting control system prototype, we attempt to cover these different approaches by considering a heuristic model to determine control actions based on user experience and with the support of smart sensors and actuators. Several heuristic models, considering artificial intelligence techniques have been reported during the last years [29], [30]. In some cases, these heuristic models seek to solve problems that traditional methods cannot work out. These solutions are often based on innovative strategies, rules or principles of data fusion. For this reason, we chose a Fuzzy logic algorithm to determine light levels required on the work plane during a working day in an office. The algorithm is treated as an additional IEEE 21451 service and was designed as shown in Figure 6.

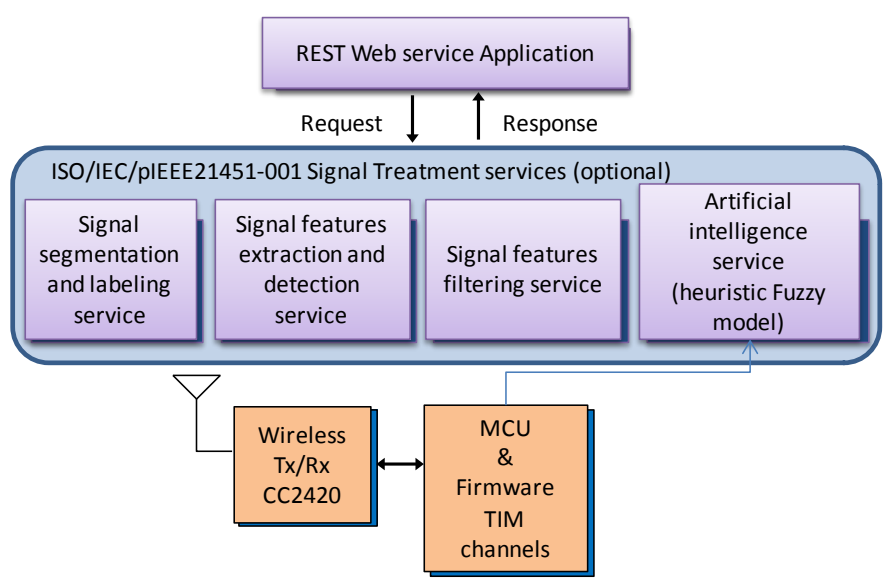

Fig 6. pIEEE21451 signal treatment services

This signal treatment service attempts to adjust lighting levels by synthesizing a set of linguistic control rules obtained from information collected during experimental measurements. The computational algorithm adjusts lighting comfort levels in compliance with UNE 12464-2012 [26]. The input variables in the Fuzzy model are occupancy, daylight level on the work plane, the position of the blinds, the schedule of work in the office and the daylight indexes which have been calculated from simulations with the ray tracing software Daysim [27]. Each input variable passes through a Mamdani Fuzzy process [28], using a logistic sigmoid function and is represented in a fuzzification process. In this case, each variable is coded as a fuzzy function and is evaluated by using certain rules of inference as shown in Table I.

TABLE I

FUZZY LOGIC ALGORITHM

\begin{tabular}{cl}
\hline \hline Step & Initialization \\
\hline 1 & $\begin{array}{l}\text { Define the linguistic variables related to illuminance } \\
\text { levels. Example: illuminance levels between (0 lx - } \\
100.000 \text { lx), very low, low, low mid, mid, high mid, high, } \\
\text { very high. }\end{array}$ \\
2 & $\begin{array}{l}\text { Construct the membership functions (trapezoidal, } \\
\text { triangular, Gaussian) } \\
\text { Construct the rules base. Example: } \\
\text { IF occupancy is on AND daylighting is very low THEN } \\
\text { artificial lighting is 100\% }\end{array}$ \\
\hline Step & Fuzzification \\
\hline 4 & $\begin{array}{l}\text { Convert input data to fuzzy values using the membership } \\
\text { functions (Mandami FIS) }\end{array}$ \\
\hline Step & Inference \\
\hline 5 & Evaluate the rules in the rule base \\
6 & Combine the results of each rule \\
\hline Step & Defuzzification \\
\hline 7 & Convert the output data to non-fuzzy values (centroid) \\
\hline \hline
\end{tabular}

The rules must be combined in some manner in order to make a decision. The algorithm uses inference rules through 
boolean logic and aggregation methods of the type (IF-ORNOT-AND) for quantifying the input variables. The output values must be defuzzified in order to resolve a single output value from the input dataset. The final desired output is the expected artificial illuminance organized as a metadata standard syntactic structure. Table II shows the syntactic message structure.

TABLE II

IEEE 21451.0 MESSAGE STRUCTURE

\begin{tabular}{|c|c|c|c|c|c|c|c|}
\hline \multicolumn{8}{|c|}{ NCAP Request } \\
\hline \multicolumn{3}{|c|}{ ZLL header } & \multicolumn{5}{|c|}{ IEEE21451.0 (header + payload) } \\
\hline $1 \mathrm{~B}$ & $2 \mathrm{~B}$ & $2 \mathrm{~B}$ & $1 \mathrm{~B}$ & $1 \mathrm{~B}$ & $1 \mathrm{~B}$ & $1 \mathrm{~B}$ & Variable \\
\hline FC & $\begin{array}{l}\text { DEV } \\
\text { ID }\end{array}$ & $\begin{array}{l}\text { CMD } \\
\text { ID }\end{array}$ & $\begin{array}{l}\text { Destination } \\
\text { Transducer } \\
\text { channel }\end{array}$ & $\begin{array}{l}\text { Cmd } \\
\text { class }\end{array}$ & $\begin{array}{l}\text { Cmd } \\
\text { Func }\end{array}$ & $\begin{array}{l}\text { Len } \\
\text { offset }\end{array}$ & payload \\
\hline \multicolumn{8}{|c|}{ TIM Response } \\
\hline \multicolumn{3}{|c|}{ ZLL header } & \multicolumn{5}{|c|}{ IEEE21451.0 (header + payload) } \\
\hline $1 \mathrm{~B}$ & $2 \mathrm{~B}$ & $2 \mathrm{~B}$ & $1 \mathrm{~B}$ & $1 \mathrm{~B}$ & & Varial & \\
\hline FC & $\begin{array}{l}\text { DEV } \\
\text { ID }\end{array}$ & $\begin{array}{l}\text { CMD } \\
\text { ID }\end{array}$ & $\begin{array}{l}\text { Flag } \\
\text { success/fail }\end{array}$ & $\begin{array}{l}\text { Length } \\
\text { offset }\end{array}$ & & paylo & \\
\hline
\end{tabular}

The headers related to the NCAP request are: ZLL Frame control (FC), ZLL device identifier (DEV ID), ZLL command identifier (CMD ID). In addition, destination transducer channel, command class (Cmd class), command function (Cmd Func), length offset and data information are the IEEE21451.0 headers.

The headers in each TIM response transaction are: ZLL Frame control, ZLL device identifier, ZLL command identifier. Also, success/fail flag, length offset and metadata payload are the IEEE21451.0 headers.

The first algorithm was initially running as a Matlab function, the NCAP sent a command to read illuminance and TIM response with a small message of 13 bytes. Then, the NCAP merged illuminance values and estimated optimized dimming values. When the output of the fuzzy algorithm obtained a response, the NCAP compared current dimming values before sending a request to change the dimming value in the TIM smart luminaire. A second algorithm runs internally in a TIM mote as an additional internal IEEE 21451 service. In this case, a Matlab Fuzzy logic FIS file is converted to a C function by using the FIST: MATLAB Fuzzy Inference System C Converter [31], and porting the code from Arduino to TinyOS 2.1. The overall compiled C-code occupies 220 bytes of RAM and 21568 bytes of ROM in the TIM nodes based on the MSP430 16-bit microcontroller.

\section{EXPERIMENTAL EVALUATION}

This section describes experimental tests that have been done to study the behavior and performance of the prototype. The first test analyzes the behavior of the prototype and its capabilities. The second test measures node autonomy based on the harvested energy. The third section presents the REST functionalities.

\section{A. IEEE 21451 compliance test}

The different capabilities of the prototype are shown in Figure 7. NCAP and TIM commands exchange is discussed in steps (1) to (7). The NCAP supports different capabilities defined as standard commands. In step 1 to 3 the NCAP creates the ZLL network and sends beacons with the aim of discovering and registering new nodes. In steps (4 -5) the TIM transducer channel accesses and reads each channel configuration. Finally, in step 6 the NCAP sends the commands corresponding to three different requests: read illuminance for monitoring purposes, set an artificial value of illuminance based on user preferences, or turn on/off the luminaire if there is occupancy detection during 15 minutes.

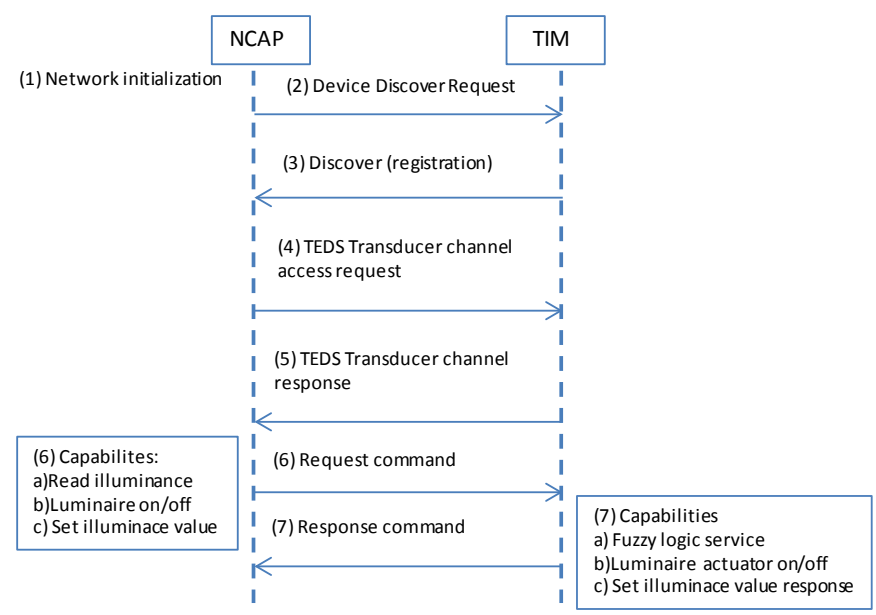

Fig 7. IEEE 21451 compliance test

In step 7, the TIM node responds to each request in different ways: for example, in case of the "read illuminance" request, it adapts the illuminance levels. The second option is to turn on/off the luminaire while a third option can set a dimming level to control the brightness from $0 \%$ to $100 \%$. We analyzed different messages captured with a docklight sniffer [32] and compared them with IEEE 21451 standard abstraction.

\section{B. Node autonomy}

Figure 8 shows a comparison between the illuminance levels close to the windows in three different working days and the energy stored in the harvester, used for supplying power to the autonomous illuminance TIM node.

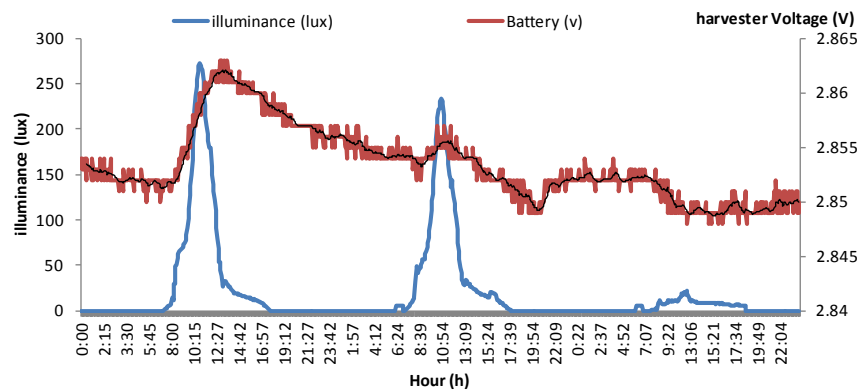

Fig 8. TIM Node autonomy test for three consecutive days (illuminance levels vs. voltage in harvester power supply)

The TIM node lifetime depends on three main factors: node location, levels of incident natural and/or artificial lighting, and ultra low duty-cycle operation. In our experimental tests, the best results were obtained with the node located near the windows in a horizontal position. Typical incident illuminance 
levels were between $0 \mathrm{~lx}$ to $290 \mathrm{~lx}$ during a 12 hour day. Dye Sensitized Solar Cells (DSSC) INDY7100 of $96 \mathrm{~cm} 2$ with an efficiency of $10 \%$ and peak wavelength sensibility in the 400 $\mathrm{nm}$ to $800 \mathrm{~nm}$ range were selected. The TIM node current consumption was measured in the three different operational modes: active, standby and sleep mode. A peak of current consumption (120 mA for $2 \mathrm{~ms}$ ) was measured when the node was reading the channel of illuminance. A second peak of 40 $\mathrm{mA}$ for $1 \mathrm{~ms}$ was obtained in Tx mode and a third peak of $25 \mathrm{~mA}$ for $520 \mathrm{~ms}$ in Rx mode. The TIM node was maintained in sleep mode during 5 minutes, consuming only $800 \mathrm{uA}$, corresponding to a duty cycle of $0.17 \%$.

\section{Graphical user interface (GUI) and REST web service}

The software application is based on a Matlab graphical user interface (GUI), as shown in Figure 9.

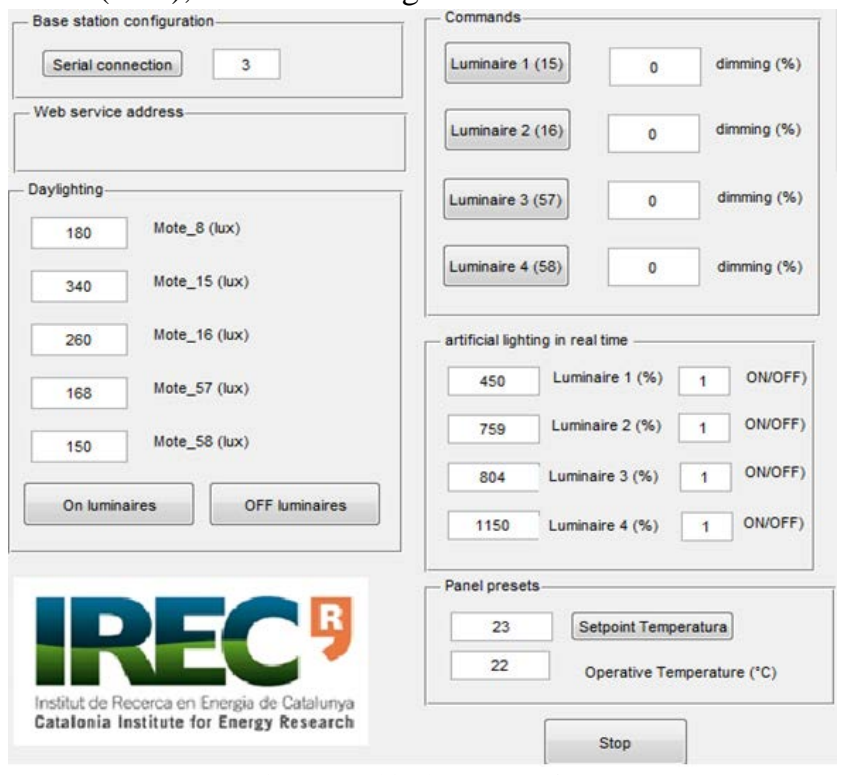

Fig 9. Graphical User Interface

The Matlab program is located on an embedded PC while information is stored in an open cloud REST web service named ThingSpeak [33].

TABLE III

FUNCTIONS TO READ THE TIM CHANNEL, TEDS AND COMMANDS

\begin{tabular}{|c|c|c|}
\hline Function & configuration & Description \\
\hline $\begin{array}{l}\text { Function [error buffer } \\
\text { num]=poll_luminaire_channel(s } \\
\text {, address, value1, value2, } \\
\text { value3, value4) } \\
\text { ZLL header: } 5 \text { bytes } \\
\text { IEEE 21451: } 4 \text { bytes } \\
\text { IEEE } 21451 \text { payload: } 4 \text { bytes }\end{array}$ & $\begin{array}{l}\text { s: NCAP } \\
\text { address: TIM } \\
\text { Value1: illuminance (lux) } \\
\text { Value2: occupancy } \\
\text { (on/off) } \\
\text { Value3: temperature (C }{ }^{\circ} \text { ) } \\
\text { Value4: energy (volts) }\end{array}$ & $\begin{array}{l}\text { Luminaire } \\
\text { command: } \\
\text { Read TIM } \\
\text { channel }\end{array}$ \\
\hline $\begin{array}{l}\text { Function [error buffer } \\
\text { num]=pollConfig(s,addr,TEDS) } \\
\text { ZLL header: } 5 \text { bytes } \\
\text { IEEE 21451: } 4 \text { bytes } \\
\text { IEEE } 21451 \text { payload: } 0 \text { bytes }\end{array}$ & $\begin{array}{l}\text { s: NCAP } \\
\text { address: TIM } \\
\text { TEDS: Configuration } \\
\text { Transducer datasheet }\end{array}$ & Read TEDS \\
\hline $\begin{array}{l}\text { Function [error buffer } \\
\text { num]=SetConfig(s,addr,TEDS_ } \\
\text { container) } \\
\text { ZLL header: } 5 \text { bytes } \\
\text { IEEE 21451: } 4 \text { bytes } \\
\text { IEEE } 21451 \text { payload: } 10 \text { bytes }\end{array}$ & $\begin{array}{l}\text { s: NCAP } \\
\text { address: TIM } \\
\text { TEDS_container: New } \\
\text { configuration }\end{array}$ & Write TEDS \\
\hline
\end{tabular}

Tables III and IV show the different message sizes and functions designed to read the TIM channel, TEDS configuration and settings corresponding to the different commands.

TABLE IV

FUNCTIONS TO READ TIM CHANNEL, TEDS CONFIGURATION AND COMMANDS

\begin{tabular}{|c|c|c|}
\hline $\begin{array}{l}\text { Function [error buffer } \\
\text { num]=dimming(s, } \\
\text { address, dimming) } \\
\text { ZLL header: } 5 \text { bytes } \\
\text { IEEE 21451: } 4 \text { bytes } \\
\text { IEEE } 21451 \text { payload: } 1 \\
\text { byte }\end{array}$ & $\begin{array}{l}\text { s: NCAP } \\
\text { address: TIM } \\
\text { Dimming: brightness } \\
\text { (\%) }\end{array}$ & $\begin{array}{l}\text { Command: set } \\
\text { dimming value }\end{array}$ \\
\hline $\begin{array}{l}\text { Function [error buffer } \\
\text { num]=changeRelay(s, } \\
\text { address, state) } \\
\text { ZLL header: } 5 \text { bytes } \\
\text { IEEE 21451: } 4 \text { bytes } \\
\text { IEEE 21451 payload: } 1 \\
\text { byte }\end{array}$ & $\begin{array}{l}\text { s: NCAP } \\
\text { address: TIM } \\
\text { State: ON/OFF }\end{array}$ & $\begin{array}{l}\text { Command: set } \\
\text { ON/OFF luminaire }\end{array}$ \\
\hline $\begin{array}{l}\text { Function [error buffer } \\
\text { num]=poll_Thermostat(s, } \\
\text { address, value1, value2) } \\
\text { ZLL header: } 5 \text { bytes } \\
\text { IEEE 21451: } 4 \text { bytes } \\
\text { IEEE } 21451 \text { payload: } 3 \\
\text { byte }\end{array}$ & $\begin{array}{l}\text { s: NCAP } \\
\text { address: TIM } \\
\text { Value1:temperature } \\
\mathrm{C}^{\circ} \text { ) } \\
{\text { Value2: humidity } \mathrm{C}^{\circ} \text { ) }}_{\text {Value3: CO2 (PPM) }}\end{array}$ & $\begin{array}{l}\text { Thermostat } \\
\text { command: Read } \\
\text { temperature,relative } \\
\text { humidity, CO2 }\end{array}$ \\
\hline
\end{tabular}

Additionally, metadata abstractions for commands are encapsulated in a JSON container that is called from Matlab to update the REST web service.

\section{User satisfaction survey}

Prior the implementation of the new lighting system, we focused on a proper work environment characterization by studying the energy consumption and the user visual comfort. Different measurements of the energy consumption, spectral reflection surfaces in the room and glare analysis with a luminance camera were taken. These data allowed us to determine experimental values and help improving the user visual comfort and sensor position, depending on the spectral contributions of natural daylight. As the old lighting system lacked dimming capabilities, the contribution factor of artificial lighting was optimized by means of a heuristic fuzzy algorithm. User satisfaction was assessed before and after installation of the new lighting system through a survey, as shown in figure 10.

A first inquiry (based on 119 responses) shows the impact of the artificial lighting and the natural lighting index on a 1 to 8 scale (1 representing very low user satisfaction, whilst 8 meaning high user satisfaction). In all cases, a user satisfaction with a score of $75 \%$ of acceptance was obtained. However, in the second survey (conducted in 2014) in the same office with the new lighting system, user acceptance was increased to $86.5 \%$. Also, energy savings were estimated by comparing the power consumption in the old lighting system to the new one. The total energy consumption for the old lighting system was 92.4 kWh (measured during April 2012). The new smart lighting system was tested between the months of December 2013 to July 2014. This system was made of the same laptop 
and PC monitor, but with a new embedded PC running a Matlab algorithm. The total energy consumption for the new system was between $52 \mathrm{kWh}$ and $80 \mathrm{kWh}$ per month.

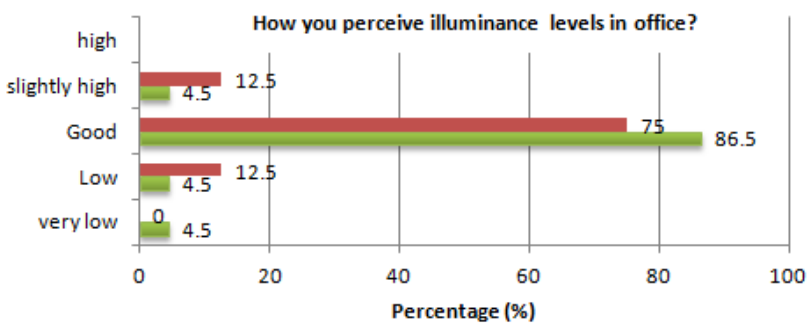

- Illuminance user perception (old system) (\%)

- Illuminance user perception (new system) (\%)

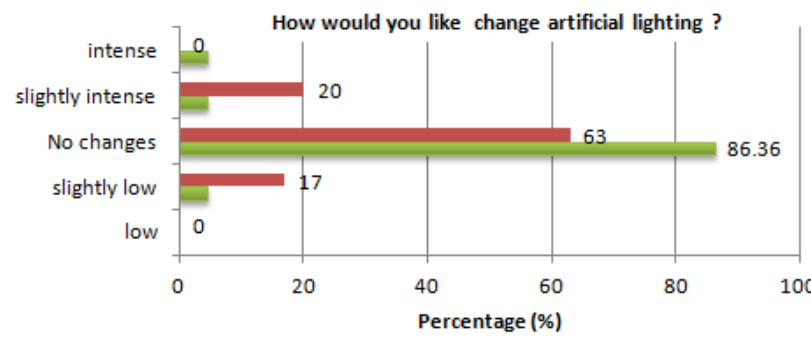

- How would you like change artificial lighting (old system) (\%)

now would you like change artificial lighting (new system) (\%)

Fig 10. Survey impact of the lighting system

Figure 11 shows a summary of the power consumption for the lighting system.

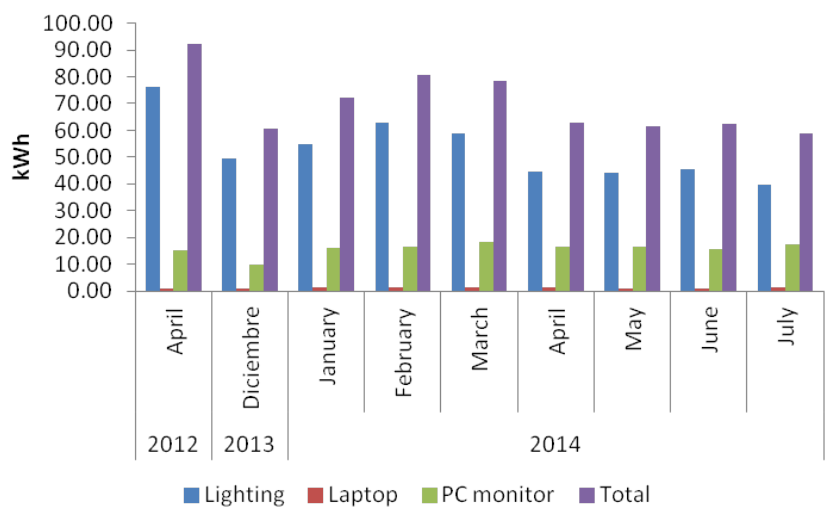

Fig 11. Power savings - smart lighting system

The energy savings have been calculated over a time period of eight months. With the new system savings between $13.4 \%$ - $43 \%$ were achieved and at the same time user satisfaction increased due to the use of a smart lighting control system. A direct comparison between April 2012 and April 2014 shows energy savings of $31.8 \%$

\section{CONCLUSIONS}

In this paper, a smart lighting system that takes into account user preferences and includes smart sensors and actuators based on IEEE 21451 is proposed. Autonomous TIM nodes provided with energy harvesting techniques were designed. A reduced set of functions that cover the core of the new IEEE 21451 standard were also implemented.

This new lighting system is able to achieve energy savings between $13 \%$ and $43 \%$ compared to the old office lighting installation. As shown in the presented quantitative survey indicators, experimental results show a reduction in operational energy costs and a clear increase in visual comfort for the occupants from $75 \%$ to $86.5 \%$.

An additional IEEE 21451 Fuzzy logic service was implemented as a Matlab function to optimize control strategies directly in the NCAP. In addition, a second IEEE 21451 fuzzy service within the TIM firmware continuously adapts dimming values to guarantee optimal visual comfort at point-of-measurement.

\section{REFERENCES}

[1] J. Byun, I Hong, B. Lee, S. Park, "Intelligent household LED lighting system considering energy efficiency and user satisfaction". IEEE Trans. on Consumer Electron., vol.59, no.1, pp.70-76, Feb. 2013.

[2] R. Srividya, C.P. Kurian. "White light source towards spectrum tunable lighting - A review", in Proc. International Conference on Advances in Energy Conversion Technologies (ICAECT), 2014, pp. 203-208

[3] Qi Chunhui; Wang Wei, "A LED Control System with Brightness and Color Temperature Adjustable", in Proc. International Conference on Control Automation and Systems Engineering (CASE), 2011, pp.1-4.

[4] D. Park, Z.Liu, H. Lee, "A 40 V 10 W 93\%-Efficiency CurrentAccuracy-Enhanced Dimmable LED Driver With Adaptive Timing Difference Compensation for Solid-State Lighting Applications", IEEE Journal of Solid-State Circuits, vol.49, no.8, pp.1848-1860, Aug. 2014.

[5] M.Martin-Arias, N.Huerta-Medina, M.Rico-Secades, "Using wireless technologies in Lighting Smart Grids", in Proc. International Conference on New Concepts in Smart Cities: Fostering Public and Private Alliances (SmartMILE), 2013, pp.1-6.

[6] F. Outeirino, F. Domingo-Perez, A. Gil-de-Castro, J. Flores, A MorenoMunoz, "In-building lighting management system with wireless communications", in Proc. IEEE International Conference on Consumer Electronics (ICCE), 2012, pp. 83-85.

[7] Min Chen, Jiafu Wan, S. Gonzalez, Xiaofei Liao; V.C.M. Leung, "A Survey of Recent Developments in Home M2M Networks", IEEE Communications Surveys \& Tutorials, vol.16, no.1, pp.98-114, First Quarter 2014

[8] Cho Hyuntae; Kim Jongdeok; Baek Yunju, "Large-scale active RFID system utilizing ZigBee networks," IEEE Transactions on Consumer Electronics, I, vol.57, no.2, pp.379-385, May 2011.

[9] ISO/IEC/IEEE Information technology -- Smart transducer interface for sensors and actuators -- Part 1: Network Capable Application Processor (NCAP) information model", ISO/IEC/IEEE 21451-1:2010(E), pp.1356, May 202010.

[10] ISO/IEC/IEEE Standard for Information technology -- Smart transducer interface for sensors and actuators -- Part 2: Transducer to microprocessor communication protocols and Transducer Electronic Data Sheet (TEDS) formats", ISO/IEC/IEEE 21451-2:2010(E), pp.1130, May 202010.

[11] ISO/IEC/IEEE Standard for Information technology -- Smart transducer interface for sensors and actuators -- Part 4: Mixed-mode communication protocols and Transducer Electronic Data Sheet (TEDS) formats", ISO/IEC/IEEE 21451-4:2010(E), pp.1-448, May 202010.

[12] Information technology--Smart transducer interface for sensors and actuators--Part 7: Transducers to radio frequency identification (RFID) systems communication protocols and transducer electronic data sheet (TEDS) formats", ISO/IEC/IEEE 21451-7:2011(E) (Revision of 1451.72010 , vol., no., pp.1,92, Feb. 102012.

[13] Lun-Wu Yeh, Che-Yen Lu, Chi-Wai Kou, Yu-Chee Tseng, Chih-Wei $\mathrm{Yi}$, "Autonomous Light Control by Wireless Sensor and Actuator Networks," IEEE Sensors Journal, vol.10, no.6, pp.1029-1041, June 2010.

[14] E.Y. Song, K.B.Lee, "Service-oriented sensor data interoperability for IEEE 1451 smart transducers", in Proc. IEEE Instrumentation and Measurement Technology Conference I2MTC, 2009, pp.1043-1048. 
[15] Sung Yong Chun; Chan-Su Lee, "Applications of Human Motion Tracking: Smart Lighting Control", in Proc. IEEE Conference on Computer Vision and Pattern Recognition Workshops (CVPRW), 2013, pp.387-392, 23-28.

[16] J.Y.Tsao, M.E.Coltrin, H.Crawford, J.A Simmons, "Solid-State Lighting: An Integrated Human Factors, Technology, and Economic Perspective", Proceedings of the IEEE , vol.98, no.7, pp.1162-1179, July 2010.

[17] I. Wang, "Zigbee light link and its applications," IEEE Wireless Communications, vol.20, no.4, pp.6-7, August 2013.

[18] R.P.V. Chander, S. Elias, S. Shivashankar, P. Manoj. "A REST based design for Web of Things in smart environments," in Proc. IEEE International Conference on Parallel Distributed and Grid Computing (PDGC), 2012, pp.337-342.

[19] K. Lee, E. Song. "Understanding IEEE 1451-Networked smart transducer interface standard - What is a smart transducer?," IEEE Instrum. \& Meas Magazine, vol.11, no.2, pp.11-17, April 2008.

[20] IEEE. P21451.1.4 Working Group (WG). Available: http://wiki.xmpp.org/web/Tech_pages/IoT_Sensei

[21] E. Callaway, P. Gorday, L. Hester, J.A Gutierrez, M. Naeve, B. Heile, V. Bahl, "Home networking with IEEE 802.15.4: a developing standard for low-rate wireless personal area networks", IEEE Communications Magazine, vol.40, no.8, pp.70-77, Aug 2002.

[22] I. Wang, "Zigbee light link and its applications," IEEE Wireless Communications, vol.20, no.4, pp.6-7, August 2013.

[23] TAOS Data sheet TSL2563, Texas Advanced Optoelectronic Solutions Inc., Plano, TX, 2009.

[24] IR-TEC Technical Data sheet MS-320LP, IR-TEC International Ltd, Luzhu, Taoyuan Taiwan, 2012.

[25] Hamamatsu Photonics Opto semiconductor products P12347-01CT, Hamamatsu Photonics K K, Hamamatsu City, pp 17, Japan, 2013.

[26] UNE-EN 12464-1:2012, "Light and lighting - Lighting of work places Part 1: Indoor work places”. UNE-EN Standard, pp 1-48, 2012.

[27] Cardoso, R.R.; Brandao, A.F., "Artificial neural network application to a building hybrid illumination system," Industrial Electronics, 2008. ISIE 2008. IEEE International Symposium on , vol., no., pp.866,871, June 30 2008-July 22008.

[28] Rocha, H.X.; Peretta, I.S.; Lima, G.F.M.; Marques, L.G.; Yamanaka, K., "Green public lighting design solved by a remote Genetic Algorithm application," Industry Applications (INDUSCON), 2012 10th IEEE/IAS International Conference on , vol., no., pp.1,6, 5-7 Nov. 2012

[29] M. González , J. Ortiz, J. Salom and J. Higuera. "Dynamic daylight simulation and visual comfort survey in Mediterranean climate. Case study in office building”, in Proc. Building Simulation Conference IBPSA, 2013, pp 1-8.

[30] A. Aceves-Lopez, J. Aguilar-Martin. "A simplified version of mamdani's fuzzy controller: the natural logic controller", IEEE Transactions on Fuzzy Systems, vol.14, no.1, pp.16-30, 2006.

[31] MAKEPROTO. MATLAB Fuzzy Inference System to C Converter. www.makeproto.com/projects/fuzzy/matlab_arduino_FIST/index.php.

[32] Flachmann \& Heggelbacher. Doclight sniffer terminal monitor Version 2.0.September 2014. http://www.docklight.de/

[33] IOBridge Inc. REST webservice Thingspeak, 2014. Available: www.thingspeak.com/channels/2504

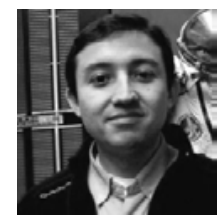

J. Higuera (M'09) received his Ph.D. in Electronic Engineering from the Universitat Politècnica de Catalunya (UPC), Barcelona, Spain, in 2011. Also, he received the B.Sc and M.Sc in Electronic Engineering from the Industrial University of Santander (UIS), Bucaramanga, Colombia in 2002 and 2006, respectively. His research interests are in the field of intelligent Lighting and interoperable wireless communications, for solid state lighting systems based on smart-sensors and actuators. He is author and co-author of several publications on international scientific journals and has served as TPC for international conferences and reviewer for journals such as the IEEE Transactions on Aerospace and Electronic Systems, IEEE Transactions on Instrumentation \& Measurement, IEEE
Transactions on Industrial Electronics and IEEE Sensors Journal.

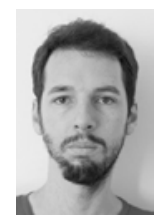

W. Hertog received his Master in Industrial Engineering: Electronics from the Catholic University College Sint-Lieven (KAHO SintLieven). After conducting research on color rendering and color perception in the Light and Lighting Laboratory of KAHO Sint-Lieven (Gent, Belgium), he started his Ph.D in the lighting group of the Catalonia Institute for Energy Research (IREC). His main research topics include intelligent lighting and spectral reproduction.

M. Perálvarez obtained his $\mathrm{PhD}$ degree (Cum Laude) and Master in Physics from University of Barcelona (UB) in 2009 and 2007, respectively. Also has obtained his bachelor degree

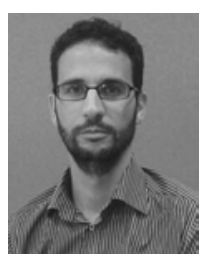
in Physics by the University of La Laguna (Canary Islands) in 2003. He is expert in the area of Si-based Optoelectronics and he has been involved in different national and European projects devoted to the fabrication, implementation and characterization of nanostructured-Si based light emitting devices. He is involved in the research and application of novel concepts for solid-state lighting with a strong focus in energy efficiency.

J. Polo received the M.E. degree in telecommunication engineering and the Ph.D. degree from the Universitat

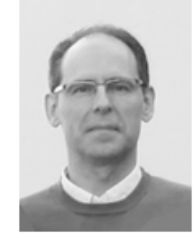
Politècnica de Catalunya (UPC), Barcelona, Spain, in 1990 and 2000, respectively. From 1993 to 1999, he was Professor with the UPC. From 1999 to 2006, he was a Professor with the Universitat Pompeu Fabra, Barcelona. Since 2006, he has been again a Professor with the UPC. Since 2007, he has authored more than 20 papers in international conferences and journals, and a regular section in a Spanish magazine about sensors. He has formed part of the organization committee of four international conferences. Initially, his research interests were electronic commerce, author right management, and services. At present, his research interests include sensor networks, mainly wireless networks, and communication standards for wireless sensor network and direct interfaces for sensors.

J. Carreras received his B.S., M.S. and PhD. (Cum Laude, with Honors) in Physics from the University of Barcelona. Dr.

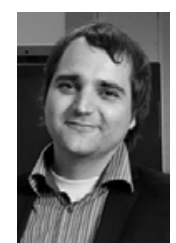
Carreras has published more than 40 articles in several SCI journals, has filed two patents, participated in more than 15 different projects at a national and international level. He is also President, founder and CTO of the spin-off Omicron Lighting. Since June 2009, he is working for Catalonia Institute for energy Research (IREC) as the Leader of the Lighting Group, leading research in novel concepts for Energy Efficient Lighting, Color Science and Technology, Simulations, Photometry, and Intelligent Lighting with advanced communication and computation functionalities. He supervises several projects in close collaboration with industrial partners. 